\title{
Introduction to theme issue on technologies for patient-defined and patient-generated data
}

\author{
Gail R. Casper · Anna McDaniel
}

Published online: 10 August 2014

(C) Springer-Verlag London 2014

Health reform initiatives around the world require active participation of individuals in their own health and in the health care process. Technologies designed to support engagement in health and health care provide the foundation for active participation of patients and engender a new vision of health services. This vision is grounded in a twoway exchange of information between patients and clinicians. Information, preferences, knowledge, and responsibility are shared to achieve the outcomes of the cooperative health efforts. Health and illness management both require more than the traditional, clinically derived signs and symptoms. They also require patient-generated data, including self-monitoring, tracking, and observations made in everyday living - the unique observations and insights that bring the everyday life of the person into the clinical encounter. Personal and ubiquitous technologies present opportunities to go beyond the traditional observation of signs and symptoms to include a complementary set of patient-generated data that include observations and activities that are centered in the patient's worldview, but sometimes defined in consultation with a clinician. In summary, we characterize three types of health data as (1) clinically defined and generated, (2) clinically defined patient-generated and (3) patient-defined and generated which we call observations of daily living or ODLs. This theme issues focuses on these last two data types, and the

G. R. Casper ( $\square)$

University of Wisconsin-Madison, Madison, WI, USA

e-mail: gcasper@wisc.edu

A. McDaniel

University of Florida, Gainesville, FL, USA

e-mail: annammcdaniel@ufl.edu majority of papers address the third-data that are both patient-defined and generated.

We advance these distinctions to foster a more comprehensive view of a person and their health concerns and their health services. Unpacking these different data types facilitates a more complete view of a person and their health experiences. Patient-defined and patient-generated data may serve as the first point of awareness and stimuli for health action. When the data are relevant and important to the patient, but not to a clinician, the patient has the opportunity—and responsibility—-to impute meaning on them.

As the realization takes hold that health occurs always and everywhere, not just during a clinic visit or a hospital stay, and as patient-generated data assume a more central role in understanding and capturing an individual patient's health state, the limits of the electronic medical record as an information platform for health emerge, and the need for newer, highly distributed nimble-but-robust information architectures becomes clearer. Achieving the goals of health for all requires full engagement in health of everyone, every day, everywhere, and this engagement, in turn, must be facilitated by knowledge and technology. The language of health care is changing, expanding to include words that reflect health-related information that enables patients to take healthy action and provides clinicians with a window into a patient's world.

Tools must be created to better support the ephemeral, idiosyncratic nature of patient-defined and patient-generated data and their episodic integration into clinical care. The challenge is twofold: (1) build tools that capture and return to the patient informative data displays, and (2) represent, package, and send these data to the clinician. Robust, powerful visualization and display strategies must be developed to accommodate the many users and uses of 
these data, from a teenager using a smartphone-based graph of exercise and intake to plan an insulin dose to the summary of that process being made available for inserting into a clinical record. Neither patients nor clinicians are likely to benefit from viewing volumes of raw data, yet both patients and clinicians can benefit from valid, trustable, and verifiable distillations and displays of the data. Distillations and display of the data have the potential to serve many purposes from self-management for patients to a patient's perspective on health for clinicians. Sharing such data between patients and clinicians has the potential to bring the everyday health lives of patients into the clinical care process. To do so efficiently requires creating a way to capture and store the data in a stable and secure manner, and to transmit that information in a timely and non-burdensome manner to the clinical team.

This theme issue brings together a set of research, design, conceptual, and methodology papers that: (1) advance our understanding of technologies that support patient engagement in their health care, (2) understand the role of technology in supporting the capture and sharing of patient-generated data, (3) document the impact of integrating patient-defined and patient-generated data into the clinical workflow and (4) stimulate advances in technologies to support this data sharing. We believe the papers that follow accomplish that and more and invite you to explore them and join the conversation. 